\title{
On the number of primitive $\lambda$-roots
}

\author{
by \\ Thomas W. Müller (London) and \\ Jan-Christoph Schlage-Puchta (Freiburg)
}

1. Introduction and results. For an integer $n$, denote by $U(n)$ the multiplicative group of residue classes modulo $n$. The structure of $U(n)$ is well known:

(i) If $n=\prod_{i=1}^{k} p_{i}^{a_{i}}$, then

$$
U(n) \cong U\left(p_{1}^{a_{1}}\right) \times U\left(p_{2}^{a_{2}}\right) \times \cdots \times U\left(p_{k}^{a_{k}}\right) .
$$

(ii) If $p$ is an odd prime, then $U\left(p^{a}\right) \cong C_{p^{a-1}(p-1)}$.

(iii) $U(2)$ is trivial, $U(4) \cong C_{2}$, and $U\left(2^{a}\right) \cong C_{2} \times C_{2^{a-2}}$ for $a \geq 3$.

The exponent of $U(n)$, that is, the least positive integer $\nu$ such that $a^{\nu} \equiv 1(\bmod n)$ for all integers $a$, is denoted by $\lambda(n)$. This function was introduced around 1910 by Carmichael; cf. [2] and [3]. By a primitive $\lambda$-root of $n$, we mean any element of maximal order $\lambda(n)$ in $U(n)$. This concept, which was introduced by Carmichael in [2], is a natural generalization of primitive roots. Let $r(n)$ be the number of primitive $\lambda$-roots of $n$. It is not difficult to see that

$$
r(n)=\varphi(n) \prod_{p \mid \varphi(n)}\left(1-p^{-m(p)}\right),
$$

where $\varphi(n)$ is Euler's totient function, and $m(p)$ is the number of elementary divisors of $U(n)$ whose $p$-part is maximal. We see that $r(n) \geq \varphi(\varphi(n))$ with equality if and only if $m(p)=1$ for all prime numbers $p$.

In [1], Cameron and Preece raise the problem to determine the density of the set

$$
\mathcal{R}=\{n: r(n)=\varphi(\varphi(n))\} .
$$

They note that a computer search reveals almost $60 \%$ of all numbers below $10^{5}$ to have this property and wonder whether the set $\mathcal{R}$ might have positive density. Integers $n \in \mathcal{R}$ have another interesting property. Define an equivalence relation $\sim$ on the set of primitive $\lambda$-roots by $a \sim b$ if and only

2000 Mathematics Subject Classification: 11N45, 11N64, 11A07. 
if $\langle a\rangle=\langle b\rangle$. Then the number of equivalence classes is at least $\varphi(n) / \lambda(n)$, with equality occurring in the latter inequality if and only if $n \in \mathcal{R}$.

For a positive integer $n$, define $f(n)$ to be the number of primes $p$ such that $m(p) \geq 2$, where $m(p)$ is defined as in (1). Our main results are as follows.

THEOREM 1. The function $f(n)$ has a normal distribution with mean $\log _{2} n / \log _{3} n$ and variance $\log _{2} n /\left(2 \log _{3} n\right)$.

TheOREM 2. For any constant $A>0$, we have

$$
\sum_{\substack{n \in \mathcal{R} \\ n \leq x}} 1 \ll \frac{x}{\left(\log _{2} x\right)^{A}}
$$

in particular, $\mathcal{R}$ has density 0 .

Here, $\log _{k} x$ denotes the $k$-fold iterated logarithm.

2. Proof of Theorem 1. We will repeatedly use the following result.

Lemma 1. Let $q \geq 3$ be an integer. Then we have uniformly in $x>e^{q}$ the estimate

$$
\sum_{\substack{p \leq x \\ p \equiv 1(\bmod q)}} \frac{1}{p} \sim \frac{\log _{2} x}{\varphi(q)} .
$$

Proof. Let $\varepsilon>0$ be given, and set $y=\exp \left((\log x)^{\varepsilon}\right)$. Using the SiegelWalfisz Theorem (see [7]), we find that

$$
\sum_{\substack{y \leq p \leq x \\ p \equiv 1(\bmod q)}} \frac{1}{p}=\frac{\log _{2} x-\log _{2} y}{\varphi(q)}+O(1),
$$

whereas the Brun-Titchmarsh-inequality (cf. [5, Theorem 3.8] or [6]) implies

$$
\sum_{\substack{q^{2} \leq p<y \\ p \equiv 1(\bmod q)}} \frac{1}{p} \leq \frac{(4+o(1)) \log _{2} y}{\varphi(q)} .
$$

Together with the trivial estimate

$$
\sum_{\substack{q \leq p<q^{2} \\ p \equiv 1(\bmod q)}} \frac{1}{p} \leq \sum_{q \leq p<q^{2}} \frac{1}{p} \ll 1
$$

our claim follows.

We now focus on the proof of Theorem 1. Note that $m(q)$ can also be described as the number of prime power block factors $p^{a}$ of $n$ such that the $q$-part of $\varphi\left(p^{a}\right)$ is maximal among all such $p$; that is, $f(n)$ is the number of prime powers $q^{a}$ satisfying the following two conditions: 
(i) there exist distinct prime divisors $p_{1}, p_{2}$ of $n$ such that $p_{1}, p_{2} \equiv 1$ $\left(\bmod q^{a}\right)$

(ii) there exists no prime divisor $p$ of $n$ such that $p \equiv 1\left(\bmod q^{a+1}\right)$.

Fix a parameter $0<\delta<1$, and define the auxiliary function $f_{\delta}(n)$ to be the number of primes $q \in\left[\delta \log _{2} n, \delta^{-1} \log _{2} n\right]$ satisfying conditions (i) and (ii). Our first aim is to show the estimate

$$
\sum_{n \leq x}\left(f(n)-f_{\delta}(n)\right) \ll \delta x \frac{\log _{2} x}{\log _{3} x} .
$$

First note that we may replace the interval $\left[\delta \log _{2} n, \delta^{-1} \log _{2} n\right]$ by $\left[\delta \log _{2} x\right.$, $\left.\delta^{-1} \log _{2} x\right]$ by increasing the value of $\delta$. Let $q^{a}$ be a prime power. We bound the number of integers $n \leq x$ such that $q^{a}$ contributes to $f(n)$ by neglecting condition (ii). This quantity equals

$$
\begin{aligned}
\sum_{\substack{p_{1}<p_{2} \\
p_{1}, p_{2} \equiv 1\left(\bmod q^{a}\right)}}\left\lfloor\frac{x}{p_{1} p_{2}}\right\rfloor & \leq \sum_{\substack{p_{1} p_{2} \leq x \\
p_{1}, p_{2} \equiv 1\left(\bmod q^{a}\right)}} \frac{x}{p_{1} p_{2}} \\
& \leq x\left(\sum_{\substack{p \leq x \\
p \equiv 1\left(\bmod q^{a}\right)}} \frac{1}{p}\right)^{2} \sim x\left(\frac{\log _{2} x}{q^{a}}\right)^{2},
\end{aligned}
$$

where we have used Lemma 1 for the last step. Summing (4) over prime power values $q^{a}>\delta^{-1} \log _{2} x$, we find that the contribution of such prime powers to the left-hand side of (3) is of acceptable magnitude. Since there are less than $\log _{2}^{1 / 2} x$ proper prime powers below $\log _{2} x$, we see that the contribution of proper prime powers is altogether negligible. Finally, there are $O\left(\delta\left(\log _{2} x\right) / \log _{3} x\right)$ prime numbers below $\delta \log _{2} x$, which is again of acceptable order, and (3) is proved.

Define $\widetilde{f}_{\delta}$ to be the number of primes $q \in\left[\delta \log _{2} x, \delta^{-1} \log _{2} x\right]$ satisfying condition (i). Then, using Lemma 1, we have

$$
\begin{aligned}
\sum_{n \leq x}\left(\widetilde{f}_{\delta}(n)-f_{\delta}(n)\right) & \leq \sum_{\delta \log _{2}} \sum_{x \leq q \leq \delta^{-1} \log _{2} x} \sum_{p \equiv 1\left(\bmod q^{2}\right)}\left\lfloor\frac{x}{p}\right\rfloor \\
& \leq x \sum_{\delta \log _{2}} \sum_{x \leq q \leq \delta^{-1} \log _{2} x} \frac{\log _{2} x}{q^{2}} \ll \frac{x}{\log _{3} x+\log \delta} .
\end{aligned}
$$

Now we use the method of moments (see, for instance, [4]) to compute the distribution of $\widetilde{f}_{\delta}$. For an integer $n$, denote by $\widetilde{m}(q)$ the number of primes $p_{i}$ satisfying condition (i). We claim that, for fixed $q \in\left[\delta \log _{2} x, \delta^{-1} \log _{2} x\right]$ and $n \in[1, x]$ chosen at random, the distribution of $\widetilde{m}(q)$ converges to a Poisson distribution with mean $\left(\log _{2} x\right) / q$, and that for different primes $q_{1}, \ldots, q_{k}$ the random variables are asymptotically independent. It follows that the 
random variables

$$
\xi_{q}= \begin{cases}1 & \text { if } \widetilde{m}(q) \geq 2 \\ 0 & \text { otherwise }\end{cases}
$$

are asymptotically independent, have means

$$
1-e^{-\left(\log _{2} x\right) / q}-\frac{\log _{2} x}{q} e^{-\left(\log _{2} x\right) / q}
$$

and variance

$$
\left(1-e^{-\left(\log _{2} x\right) / q}-\frac{\log _{2} x}{q} e^{-\left(\log _{2} x\right) / q}\right)\left(e^{-\left(\log _{2} x\right) / q}+\frac{\log _{2} x}{q} e^{-\left(\log _{2} x\right) / q}\right) .
$$

From this, Theorem 1 follows in view of the facts that

$$
\begin{aligned}
\int_{0}^{\infty}\left(1-e^{-1 / t}-\frac{1}{t} e^{-1 / t}\right) d t & =1, \\
\int_{0}^{\infty}\left(1-e^{-1 / t}-\frac{1}{t} e^{-1 / t}\right)\left(e^{-1 / t}+\frac{1}{t} e^{-1 / t}\right) d t & =\frac{1}{2} .
\end{aligned}
$$

Hence, it remains to study the higher moments of the variables $\widetilde{m}(q)$ and their correlations. To do so, we compute the expected value of $\left(\begin{array}{c}\widetilde{m}(q) \\ k\end{array}\right)$ for fixed $k \geq 1$. We find that

$$
\begin{aligned}
\mathbf{E}\left(\begin{array}{c}
\tilde{m}(q) \\
k
\end{array}\right) & =\sum_{n \leq x}\left|\left\{p_{1}<\cdots<p_{k}: p_{i} \equiv 1(\bmod q), p_{i} \mid n\right\}\right| \\
& =\sum_{\substack{p_{1}<\cdots<p_{k} \\
p_{i} \equiv 1(\bmod q)}}\left|\frac{x}{p_{1} \cdots p_{k}}\right|=\sum_{\substack{p_{1}<\cdots<p_{k} \\
p_{i} \equiv 1(\bmod q) \\
p_{1} \cdots p_{k} \leq x}} \frac{x}{p_{1} \cdots p_{k}}+O\left(\frac{x \log _{2}^{k} x}{\log x}\right) \\
& =\frac{x}{k !}\left(\sum_{\substack{p \leq x \\
p \equiv 1(\bmod q)}} \frac{1}{p}+O\left(\frac{1}{q}\right)\right)^{k}+O\left(\frac{x}{\log _{2} x}\right) \\
& =\frac{x}{k !}\left(\frac{\log _{2} x}{q}\right)^{k}+O\left(\frac{x}{\log _{2} x}\right) .
\end{aligned}
$$

On the other hand, the $k$ th moment of a Poisson distribution with mean $\left(\log _{2} x\right) / q$ is

$$
\mathbf{E}\left(\xi^{k}\right)=\sum_{\kappa=0}^{k} S_{\kappa, k}\left(\frac{\log _{2} x}{q}\right)^{\kappa},
$$

where the $S_{\kappa, k}$ are Stirling numbers of the second kind. By the Stirling 
inversion formula, the last assertion is equivalent to

$$
\sum_{\kappa=0}^{k} s_{\kappa, k}\left(\frac{\log _{2} x}{q}\right)^{\kappa}=\left(\frac{\log _{2} x}{q}\right)^{k}
$$

where the $s_{\kappa, k}$ are Stirling numbers of the first kind. Since

$$
\sum_{\kappa=0}^{k} s_{\kappa, k} x^{\kappa}=x(x-1) \cdots(x-k+1),
$$

the variables $\widetilde{m}(q)$ converge to a Poisson distribution with mean $\left(\log _{2} x\right) / q$.

To show that the variables $\widetilde{m}(q)$ are asymptotically independent, it suffices to show that for fixed integers $k_{1}, \ldots, k_{l}$, we have

$$
\mathbf{E}\left(\begin{array}{c}
\tilde{m}\left(q_{1}\right) \\
k_{1}
\end{array}\right) \cdots\left(\begin{array}{c}
\tilde{m}\left(q_{l}\right) \\
k_{l}
\end{array}\right) \sim\left(\mathbf{E}\left(\begin{array}{c}
\tilde{m}\left(q_{1}\right) \\
k_{1}
\end{array}\right)\right) \cdots\left(\mathbf{E}\left(\begin{array}{c}
\tilde{m}\left(q_{l}\right) \\
k_{l}
\end{array}\right)\right) .
$$

The left-hand side quantity can be written as

$$
\sum_{n \leq x}\left|\left\{p_{11}<\cdots<p_{1 k_{1}}, \ldots, p_{l 1}<\cdots<p_{l k_{l}}: \forall i, j: p_{i j} \equiv 1\left(\bmod q_{i}\right), p_{i j} \mid n\right\}\right| \text {. }
$$

If all primes $p_{i j}$ are different, this can be computed as above and is easily seen to be asymptotically equal to the right-hand side of (5). It suffices to compare the contribution of tuples satisfying $p_{11}=p_{21}$, say, with all tuples. Note that restricting $p_{i j}$ by $x^{1 /(2 k)}$ does not change the expectations significantly, hence, writing $M$ for the least common multiple of all $p_{i j}$, $(i, j) \neq(1,1),(1,2)$, we have $M \leq \sqrt{x}$. Then we obtain

$$
\sum_{\substack{n \leq x \\ M \mid n}} \sum_{\substack{p \mid n \\ p \equiv 1\left(\bmod q_{1} q_{2}\right)}} 1 \ll \frac{x \log _{2} x}{M q_{1} q_{2}}+m \frac{x}{M},
$$

where $m$ denotes the number of primes among $p_{i j},(i, j) \neq(1,1),(1,2)$, which are congruent to 1 modulo $q_{1} q_{2}$. Since

$$
\sum_{\substack{n \leq x \\ M \mid n}}\left|\left\{p_{1} \equiv 1\left(\bmod q_{1}\right), p_{2} \equiv 1\left(\bmod q_{2}\right), p_{1}, p_{2} \mid n\right\}\right| \gg \frac{x \log _{2}^{2} x}{M q_{1} q_{2}}+m \frac{x}{M},
$$

we see that tuples with repetitions are indeed negligible, proving that the random variables $\widetilde{m}(q)$ are asymptotically independent.

3. Proof of Theorem 2. Define $f_{\delta}$ as in the proof of Theorem 1. Since $f(n) \geq f_{\delta}(n)$, it suffices to consider the set

$$
\mathcal{R}_{\delta}:=\left\{n: f_{\delta}(n)=0\right\} .
$$

Moreover, from the computation of the moments of $\widetilde{f}_{\delta}$ we know that the number of integers $n \leq x$ satisfying $\widetilde{f}_{\delta}(n) \leq \frac{1}{2} \log _{2} x$ is bounded above 
by $O\left(x / \log _{2}^{A} x\right)$ for every constant $A$, provided that $\delta$ is sufficiently small. Hence, it suffices to consider the set

$$
\mathcal{S}_{\delta}:=\left\{n: \widetilde{f}_{\delta}(n)-f_{\delta}(n) \geq \frac{1}{2} \log _{2} x\right\} .
$$

For an integer $k \geq 1$, we have

$$
\begin{aligned}
& \sum_{n \leq x}\left(\begin{array}{c}
\tilde{f}_{\delta}(n)-f_{\delta}(n) \\
k
\end{array}\right) \\
& \leq \sum_{\delta \log _{2} x \leq q_{1}<\cdots<q_{k} \leq \delta^{-1} \log _{2} x}\left|\left\{\left(n, p_{1}, \ldots, p_{k}\right): p_{i} \mid n, p_{i} \equiv 1\left(\bmod q_{i}^{2}\right)\right\}\right| .
\end{aligned}
$$

Restricting the range for $p_{i}, 1 \leq i \leq k$, to $\left[1, x^{1 /(2 k)}\right]$ introduces an error term of order

$$
\sum_{\delta \log _{2} x \leq q_{1}<\cdots<q_{k} \leq \delta^{-1} \log _{2} x} \frac{1}{q_{1}^{2} \cdots q_{k}^{2}} \ll \delta^{-k} \log _{2}^{-k} x .
$$

Now fix $q_{1}, \ldots, q_{k}$ as above, and assume that $p_{1}=p_{2}$, say. Fix $p_{3}, \ldots, p_{k}$, and let $M$ be the least common multiple of $p_{3}, \ldots, p_{k}$. Then the contribution of all possible choices for $p_{1}$ and $p_{2}$ is

$$
\left|\left\{(n, p): p M \mid n, p \equiv 1\left(\bmod q_{1}^{2} q_{2}^{2}\right)\right\}\right| \leq(1+o(1)) \frac{x \log _{2} x}{M q_{1}^{2} q_{2}^{2}},
$$

whereas the number of all triples $\left(n, p_{1}, p_{2}\right)$ is $(1+o(1))\left(x \log _{2}^{2} x\right) /\left(M q_{1}^{2} q_{2}^{2}\right)$. Hence, the contribution of tuples $\left(n, p_{1}, \ldots, p_{k}\right)$ with repetitions to the righthand side of (6) is of lesser order than the contribution of tuples without repetitions. We obtain

$$
\begin{gathered}
\sum_{n \leq x}\left(\begin{array}{c}
\tilde{f}_{\delta}(n)-f_{\delta}(n) \\
k
\end{array}\right) \\
\leq(1+o(1)) x \sum_{\delta \log _{2} x \leq q_{1}<\cdots<q_{k} \leq \delta^{-1} \log _{2} x} \prod_{i=1}^{k}\left(\sum_{\substack{p \leq x \\
p \equiv 1\left(\bmod q_{i}^{2}\right)}} \frac{1}{p}\right) \\
\leq(1+o(1)) x \sum_{\delta \log _{2} x \leq q_{1}<\cdots<q_{k} \leq \delta^{-1} \log _{2} x} \frac{\log _{2}^{k} x}{q_{1}^{2} \cdots q_{k}^{2}} \\
\leq \frac{(1+o(1)) x\left(\pi\left(\delta^{-1} \log _{2} x\right)\right)^{k}}{\delta^{2 k} \log _{2}^{k} x} \leq \frac{(1+o(1)) x}{\delta^{3 k} \log _{3}^{k} x} .
\end{gathered}
$$

Since integers $n$ with $\widetilde{f}_{\delta}(n)-f_{\delta}(n) \geq \frac{1}{2} \log _{2} x$ contribute at least $\left(\log _{2}^{k} x\right) / 3^{k} k$ ! to the left-hand side of (7), Theorem 2 follows. 


\section{References}

[1] P. J. Cameron and D. A. Preece, Notes on primitive lambda-roots, manuscript, available as http://www.maths.qmul.ac.uk/ pjc/csgnotes/lambda.pdf.

[2] R. D. Carmichael, Note on a new number theory function, Bull. Amer. Math. Soc. 16 (1909-1910), 232-238.

[3] -, Generalizations of Euler's $\phi$-function, with applications to Abelian groups, Quart. J. Math. 44 (1913), 94-104.

[4] P. D. T. A. Elliott, Probabilistic Number Theory II. Central Limit Theorems, Grundlehren Math. Wiss. 240, Springer, Berlin, 1980.

[5] H. Halberstam and H.-E. Richert, Sieve Methods, Academic Press, London, 1974.

[6] H. L. Montgomery and R. C. Vaughan, On the large sieve, Mathematika 20 (1973), 119-134.

[7] C. L. Siegel, Über die Classenzahl quadratischer Zahlkörper, Acta Arith. 1 (1936), 83-86.

School of Mathematical Sciences

Queen Mary

University of London

Mathematisches Institut

Mile End Road

Albert-Ludwigs-Universität

Eckerstr. 1

E1 4NS London, UK

79104 Freiburg, Germany

E-mail: T.W.Muller@qmul.ac.uk

Received on 22.9.2003

and in revised form on 19.12.2003 